\title{
Henry per Meter
}

National Cancer Institute

\section{Source}

National Cancer Institute. Henry per Meter. NCI Thesaurus. Code C68913.

A SI unit of magnetic permeability equal to one kilogram meter per second squared per ampere squared. 